\title{
Estímulo ao desenvolvimento infantil: produção do conhecimento em Enfermagem
}

\author{
Stimulus to child development: knowledge production in nursing
}

Estímulo al desarrollo infantil: producción de conocimiento en Enfermería

\author{
Bruna Cristine Peres Falbo', Raquel Dully Andrade", \\ Maria Cândida de Carvalho Furtado"', Débora Falleiros de Mello"' \\ ' Universidade de São Paulo, Escola de Enfermagem de Ribeirão Preto, \\ Curso de Bacharelado em Enfermagem. (Graduanda) Ribeirão Preto-SP, Brasil. \\ "Universidade de São Paulo, Escola de Enfermagem de Ribeirão Preto, \\ Programa de Pós-Graduação em Enfermagem. (Doutoranda) Ribeirão Preto-SP, Brasil. \\ II' Universidade de São Paulo, Escola de Enfermagem de Ribeirão Preto, \\ Departamento de Enfermagem Materno Infantil e Saúde Pública. Ribeirão Preto-SP, Brasil.
}

Submissão: 16-11-2010 Aprovação: 27-12-2011

\section{RESUMO}

Este estudo teve como objetivo identificar as ações de enfermagem para estímulo ao desenvolvimento infantil, em periódicos nacionais e internacionais, no período de 2000 a 2009. Revisão integrativa da literatura, nas bases de dados Medline e Lilacs, com análise de quinze artigos. Os resultados apontam o lúdico como essencial ao desenvolvimento infantil, devendo ser explorado pela enfermagem por meio da arte, música, brinquedos e teatro. Amenizar o estresse da hospitalização com adequação ambiental reduz o seu impacto sobre o desenvolvimento infantil. Orientar e intervir nos inter-relacionamentos são ações de enfermagem para o desenvolvimento social e comportamental infantil. Os temas abordados na literatura são fundamentais para uma prática de enfermagem no cuidado integral à saúde da criança.

Descritores: Criança; Desenvolvimento infantil; Enfermagem.

\begin{abstract}
This integrative literature review aimed to identify nursing actions for stimulating child development in national and international journals, between 2000 and 2009. Medline and Lilacs were examined, with analysis of fifteen articles. Results point out ludic elements as essential for child development and state that it should be explored by nursing professionals through art, music, toys and theatre. Soften the stress of hospitalization through environmental adjustments reduces its impact on child development. Guiding and intervening in the inter-relationships are nursing actions for the social and behavioral development of children. The themes addressed in literature are essential to the nursing practice in comprehensive child care.
\end{abstract}

Key words: Child; Child development; Nursing.

\section{RESUMEN}

Esta revisión integradora de la literatura tuvo como objetivo identificar las acciones de enfermería para el estímulo del desarrollo infantil, en artículos publicados en revistas brasileñas e internacionales entre 2000 y 2009. Los artículos estaban disponibles en las bases de datos Medline y Lilacs. Fueron analizados quince artículos. Los resultados señalan el lúdico como esencial para el desarrollo infantil y debe ser explorado por la enfermería a través del arte, música, juguetes y teatro. Aliviar el estrés de la hospitalización por medio de la adecuación ambiental reduce el impacto en el desarrollo infantil. Guiar e intervenir en las interrelaciones son acciones de enfermería para el desarrollo social y del comportamiento infantil. Los temas tratados en la literatura son fundamentales para la práctica de enfermería en la atención integral a la salud infantil.

Palabras clave: Niño; Desarrollo infantil; Enfermería. 


\section{INTRODUÇÃO}

Na saúde infantil vêm ocorrendo avanços no tocante à redução da mortalidade infantil e ampliação da cobertura dos serviços de saúde, enfatizando-se que não basta a sobrevivência das crianças, mas que é preciso oferecer condições para que as mesmas vivam com qualidade, permitindo o desenvolvimento de seu potencial e o usufruto de bens que a sociedade produz.

As Metas para o Desenvolvimento do Milênio implicam em compromissos por todos os países em relação ao desenvolvimento global das sociedades, tendo como principais desafios: promover a redução da pobreza, estimular a educação, a saúde materna, a igualdade de gênero, bem como o propósito de combater e reduzir a mortalidade infantil, na perspectiva de aliança global(1).

No Brasil, a saúde da criança tem enfoque no acompanhamento do crescimento e desenvolvimento infantil, incentivo ao aleitamento materno, orientação da alimentação da criança, imunização, prevenção de acidentes e atenção às doenças prevalentes na infância. Essas práticas de saúde constituem elementos essenciais para proporcionar boas condições de saúde na infância(2).

O acompanhamento do crescimento e do desenvolvimento infantil deve ser feito de forma regular, de modo que seja possível a detecção precoce de alterações, viabilizando as devidas condutas em tempo hábil, com o objetivo de proporcionar à criança oportunidades para um desenvolvimento adequado durante toda a infância, contribuindo para que suas potencialidades sejam desenvolvidas, de forma a refletir positivamente por toda a vida ${ }^{(3)}$. No Brasil, o principal agente, responsável por esse acompanhamento nos serviços de atenção primária à saúde, tem sido o enfermeiro.

O desenvolvimento infantil, nas últimas décadas, em termos mundiais, tem sido tema de interesse, como resultado do aumento constante da sobrevivência infantil e do reconhecimento de que a prevenção de problemas ou de patologias exerce efeitos duradouros na constituição do ser humano ${ }^{(4)}$. Nesse sentido, o desenvolvimento infantil tem sido estudado, salientando a relação entre pobreza e inadequada criação das crianças, apontando problemas na estrutura familiar, hostilidade e desinteresse dos pais, depressão materna, ausência de cuidadores e de um ambiente de suporte ${ }^{(5)}$.

O enfermeiro, quando atua junto à clientela infantil, deve considerar as diversas dimensões inter-relacionadas à saúde das crianças às quais assiste, afastando-se de uma intervenção restrita à clínica biológica apresentada.

Dentro dessa perspectiva, o desenvolvimento da criança tem sido enfatizado e valorizado no contexto da família. As relações parentais podem estar relacionadas a problemas no desenvolvimento cognitivo, social e do desempenho escolar da criança ${ }^{(6)}$. O vínculo é importante fator de proteção para o desenvolvimento, da mesma forma que uma relação desestruturada na família pode ser um fator de influência negativa ${ }^{(3)}$. Estudos mostram que a relação de apego, principalmente no primeiro ano de vida, está intimamente ligada ao desenvolvimento afetivo, cognitivo e social da criança ${ }^{(7-9)}$. Estimular esse vínculo é um papel importante da equipe de enfermagem.

O acompanhamento do desenvolvimento deve ser realizado na atenção primária à saúde, e deve contar com o apoio da família, comunidade e profissionais da saúde. Cabe ao enfermeiro deter o conhecimento necessário para avaliação da criança, tomada de decisões e orientação da família. O enfermeiro deve compreender a infância e os seus diferentes contextos de desenvolvimento, incluindo desde o micro-sistema familiar até o macro-sistema da cultura em que a criança se insere.

Estas ações são importantes para que a criança possa receber atenção à saúde adequada e contextualizada ao seu meio e condições de vida, estímulo ao cuidado e atendimentos específicos às suas necessidades, visando ao seu desenvolvimento saudável. Assim, a enfermagem deve adotar estratégias de promoção e prevenção para intervir o mais precocemente, ressaltando a responsabilidade de disponibilizar uma assistência à saúde qualificada e humanizada e de favorecer o desenvolvimento humano.

Muitas das questões relacionadas ao tema do desenvolvimento humano vêm sendo discutidas na atualidade, tornando-se importante ampliar as discussões sobre essa temática na área da Enfermagem, com vistas a contribuir para as reflexões do cuidado integral à saúde da criança. Este estudo teve como objetivo identificar as ações de enfermagem para estímulo ao desenvolvimento infantil, em periódicos nacionais e internacionais, no período de 2000 a 2009.

\section{PERCURSO METODOLÓGICO}

Esta investigação configura-se como uma revisão integrativa da literatura, a partir de referências bibliográficas da área da Enfermagem sobre ações que promovem e estimulam o desenvolvimento infantil.

A revisão integrativa da literatura é considerada um instrumento da prática baseada em evidências (PBE), e tem o potencial de construir conhecimento fundamentado e uniforme para a realização de uma prática clínica de qualidade ${ }^{(10)}$. As etapas que compõem esta revisão integrativa foram: identificação do tema e seleção da questão de pesquisa, busca nas bases de dados digitais, estabelecimento de critérios para inclusão e exclusão de estudos, busca dos textos na íntegra, definição das informações a serem extraídas dos estudos selecionados, categorização e avaliação dos estudos incluídos, interpretação dos resultados e apresentação da revisão e síntese do conhecimento ${ }^{(10)}$.

A questão norteadora adotada para esse estudo foi: qual é o conhecimento científico produzido pela Enfermagem acerca das ações que promovem e estimulam o desenvolvimento infantil? Para a seleção dos artigos foram utilizadas as palavras-chave: desenvolvimento infantil, promoção da saúde, atenção primária e enfermagem, junto às bases de dados Medline e Lilacs.

A tabela 1 apresenta os resultados encontrados nas bases consultadas, com o número de referências bibliográficas obtidas em cada uma e o número de referências selecionadas, por abrangerem a questão norteadora. 
Tabela 1 - Distribuição de referências bibliográficas obtidas nas bases de dados de acordo com as palavras-chave estabelecidas.

\begin{tabular}{cccc}
\hline Base de dados & Palavras Chave & Referências encontradas & Referências selecionadas \\
\hline Lilacs & desenvolvimento infantil promoção da saúde atenção primária & 3 & 0 \\
Lilacs & desenvolvimento infantil enfermagem atenção primária & 1 & 0 \\
Lilacs & desenvolvimento infantil enfermagem promoção da saúde & 4 & 1 \\
Lilacs & desenvolvimento infantil enfermagem & 43 & 0 \\
Lilacs & desenvolvimento infantil atenção primária & 25 & 2 \\
Lilacs & desenvolvimento infantil promoção da saúde & 38 & 1 \\
Medline & desenvolvimento infantil enfermagem & 3 & 0 \\
Medline & desenvolvimento infantil promoção da saúde nursing & 2 & 2 \\
Medline & desenvolvimento infantil promoção da saúde nursing & 6 & 7 \\
Medline & desenvolvimento infantil promoção da saúde atenção primária & 0 & 0 \\
Medline & desenvolvimento infantil atenção primária nursing & 2 & 0 \\
Medline & child development nursing & $\mathbf{1 5 7}$ & $\mathbf{1 6}$ \\
\hline
\end{tabular}

Por meio do cruzamento das palavras-chave, foram encontrados 157 artigos. Após uma leitura criteriosa dos resumos para averiguar se contemplava a questão norteadora, 16 estudos foram selecionados, que atendiam tanto à questão norteadora quanto aos critérios de inclusão. Desses estudos, um se repetiu nas duas bases de dados, portanto 15 artigos compõem esta revisão.

Os trabalhos incluídos na presente revisão obedeceram aos seguintes critérios de inclusão: resumo disponível nas bases de dados descritas; idioma de publicação português, inglês ou espanhol; período de publicação compreendido entre os anos 2000 e 2009 e com temáticas que envolvem a promoção do desenvolvimento infantil e a enfermagem. Foram consideradas publicações com todos os tipos de delineamentos e evidências científicas, devido à característica de nossa questão norteadora, que não se relaciona à eficácia de uma intervenção, mas sim à abrangência do conhecimento produzido acerca da temática. A incorporação de vários tipos de estudos em revisões é extremamente importante, visando uma melhor contribuição para diferentes situações da prática profissional ${ }^{(11)}$.

Após a análise dos textos na íntegra, foi realizada uma síntese dos dados, contemplando título, autores, objetivo, metodologia, resultados e conclusões. A seleção e análise dos estudos foram realizadas por duas pesquisadoras e confrontadas posteriormente, sendo selecionados e analisados, ao todo, 15 artigos. A análise dos dados foi realizada de forma descritiva, procedendo-se à categorização das informações extraídas dos estudos selecionados em grupos temáticos, a partir da identificação de aspectos relevantes de interesse para o foco do estudo, conforme proposto em literatura específica acerca de revisão integrativa de literatura ${ }^{(12)}$.

\section{RESULTADOS E DISCUSSÃO}

A tabela 2 mostra dados da distribuição dos estudos incluídos na pesquisa de acordo com as bases de dados, ano e país de publicação, autores, área de atuação e metodologia.

Durante o período de 2005 a 2009, o ano que apresentou um maior número de trabalhos publicados com a temática em foco foi o de 2005 (4 artigos). A base de dados que forneceu um número maior de trabalhos foi o Lilacs (10 artigos), enquanto os países de origem das pesquisas selecionadas foram Brasil (10 artigos) e Estados Unidos (4 artigos). Observa-se que o Brasil tem se destacado na produção de estudos de enfermagem sobre ações de estímulo ao desenvolvimento infantil na última década. Isso pode estar relacionado ao fato de o desenvolvimento infantil ser um dos enfoques das ações de saúde da criança no Brasil (2).

Observa-se uma concentração de estudos descritivos, com 13 artigos. Entre esses, 9 usaram a abordagem qualitativa e 4 a quantitativa, para análise dos dados. Dois trabalhos configuravam-se como revisão de literatura. Identifica-se uma ausência de estudos de intervenção que tratem das ações de enfermagem para estímulo do desenvolvimento infantil.

A análise de aspectos relevantes, de interesse para o foco do estudo, nos artigos selecionados, permitiu a apresentação de três temas: O lúdico e o desenvolvimento infantil, O ambiente hospitalar e o desenvolvimento infantil, Os inter-relacionamentos e o desenvolvimento infantil. Estas unidades temáticas são apresentadas a seguir.

\section{O lúdico e o desenvolvimento infantil}

O brincar é fundamental para o crescimento e desenvolvimento adequados da criança. O lúdico, destacado pela música, teatro, jogos e brincadeiras, auxilia no reconhecimento, enfrentamento e adaptação da criança ao mundo que a cerca. Brincando a criança conhece, explora e compreende o mundo, aperfeiçoa seu poder de comunicação e interação com outras crianças ${ }^{(13)}$. Brincar estimula o desenvolvimento físico, psicológico, social e moral, e auxilia no aperfeiçoamento das habilidades psicomotoras ${ }^{(13-15)}$.

Para a criança hospitalizada, estudos apontam que brincar tem valor terapêutico, pois permite que ela libere seus 
Tabela 2 - Distribuição de referências bibliográficas de acordo com as bases de dados, ano e país de publicação, autores, área de atuação e tipo de estudo.

\begin{tabular}{ccccccc}
\hline & Ano & Base de dados & Origem & Autor & Área de atuação & Tipo de estudo \\
\hline 1 & 2000 & Medline & EUA & Harrison et al. & Enfermagem & Estudo descritivo qualitativo \\
2 & 2002 & Lilacs/Medline & Brasil & Paula et al. & Enfermagem & Estudo descritivo qualitativo \\
3 & 2003 & Lilacs & Brasil & Rezende et al. & Enfermagem & Estudo descritivo quantitativo \\
4 & 2005 & Lilacs & Brasil & Ravelli et al. & Enfermagem & Estudo descritivo qualitativo \\
5 & 2005 & Lilacs & Brasil & Regis et al. & Enfermagem e psicologia & Estudo descritivo qualitativo \\
6 & 2005 & Lilacs & Brasil & Rezende et al. & Enfermagem & Estudo descritivo quantitativo \\
7 & 2005 & Lilacs & Brasil & Valladares, Pimenta. & Enfermagem e arteterapia & Estudo descritivo quantitativo \\
8 & 2006 & Lilacs & Brasil & Valladares et al. & Enfermagem e arteterapia & Estudo descritivo quantitativo \\
9 & 2006 & Lilacs & Brasil & Silva et al. & Enfermagem & Revisão da literatura \\
10 & 2007 & Medline & EUA & Stouffer et al. & Enfermagem & Estudo descritivo qualitativo \\
11 & 2007 & Medline & EUA & Nehring, W. M. & Enfermagem & Estudo descritivo qualitativo \\
12 & 2008 & Lilacs & Brasil & Magnabosco et al & Enfermagem & Revisão da literatura \\
13 & 2008 & Lilacs & Brasil & Bortolote et al. & Enfermagem & Estudo descritivo qualitativo \\
14 & 2008 & Medline & EUA & Mansell et al. & Enfermagem & Estudo descritivo qualitativo \\
15 & 2009 & Lilacs & Brasil & Lima et al. & Enfermagem & Estudo descritivo qualitativo \\
\hline
\end{tabular}

temores, raivas, frustrações e ansiedades, além de facilitar a comunicação com a equipe de saúde ${ }^{(14,16-17)}$. O lúdico oferece à criança momentos para expressar seus sentimentos oriundos da doença e da internação e promove a manutenção de uma relação saudável entre a criança, sua família e a equipe de saúde ${ }^{(14-15)}$.

O hospital pode não ser um ambiente de dor e sofrimento quando seu espaço é aproveitado também para o desenvolvimento de atividades lúdicas, pedagógicas e recreacionais. Nesse sentido, tem sido explorado o teatro clown, inserido no contexto da hospitalização, que oferece oportunidade de uma nova forma de comunicação com a criança, que permite que ela exteriorize seus medos, dores, angústias e limitações, além de estimular o riso e a imaginação ${ }^{(14)}$. A partir da observação dos pesquisadores e relato de mães, o teatro clown foi visto como uma estratégia que distrai as crianças e minimiza o estresse da hospitalização ${ }^{(14)}$.

O Teatro Clown caracteriza-se por uma representação cômica da realidade, por meio de uma arte que possui um grande potencial lúdico, utilizando como estímulo um pensamento crítico sobre o próprio "eu". O exercício dessa técnica pode ter alto potencial benéfico entre pessoas que estão vivenciando momentos de vulnerabilidade e sofrimento, com sobrecarga emocional e desgaste psíquico, o que ocorre especialmente com crianças hospitalizadas e seus acompanhantes, podendo contribuir para a capacidade de enfretamento.

Durante a hospitalização, a arteterapia se demonstrou como uma modalidade lúdica que incentiva o desenvolvimento normal da criança ${ }^{(17-18)}$. A criança, por meio da arte, reduz a confusão cognitiva provocada pela hospitalização, possibilitando o descobrimento de potencialidades e a ampliação do universo pessoal, recontextualizando antigos bloqueios que adquirem novos significados ${ }^{(17-18)}$. A arte possibilita a liberdade de expressão, sustenta a autonomia criativa, promove o desenvolvimento da atenção, concentração, coordenação, bem como concretiza pensamentos e exercita a memória ${ }^{(18)}$.

Um estudo demonstra que a música, presente na vida da criança desde o ambiente intra-útero, contribui para o período da infância, destacando as cantigas de ninar que acalentam o sono dos recém-nascidos e fortalecem o vínculo afetivo entre criança e cuidador, enquanto as cantigas de roda na fase pré-escolar estimulam a interação com outras crianças ${ }^{(15)}$. A música é considerada um valioso método de distração e pode ser empregada como instrumento no tratamento de inúmeras doenças e no alívio da dor, pois tem o poder de suavizar tensões, reduzir a ansiedade e provocar melhora no padrão de sono e humor ${ }^{(14,19)}$. Quando utilizada como terapia, a música deve ser selecionada criteriosamente de acordo com o nível desenvolvimental da criança, para que os objetivos terapêuticos sejam alcançados ${ }^{(19)}$. A música é um estímulo para a criança cantar e movimentar-se, já que muitas canções incluem gestos e movimentos corporais ${ }^{(14-15)}$, sendo mais uma forma para comunicar-se com o mundo ${ }^{(20)}$.

O cuidado lúdico propicia o autoconhecimento e conhecimento do outro com prazer e descontração, favorece a socialização das experiências e dos valores. Através do cuidado lúdico é possível conhecer a criança e, assim, estimular o desenvolvimento de suas potencialidades. A enfermagem deve embarcar no mundo lúdico da criança para poder compreendê-la e oferecer um cuidado diferenciado, eficaz e humanizado. Os profissionais de enfermagem, agentes que desempenham o papel de facilitadores do crescimento e do desenvolvimento infantil, devem conhecer a criança no seu contexto social e familiar, para despertar seus potenciais ${ }^{(13)}$.

Considera-se importante que seja oportunizado ao enfermeiro que atua junto à clientela infantil e sua equipe, a 
participação em atividades educativas permanentes que capacitem e estimulem o uso de estratégias e ferramentas lúdicas na interação com a criança.

\section{O ambiente hospitalar e o desenvolvimento infantil}

Quando uma criança necessita de hospitalização ela é afastada do seu ambiente habitual - sua casa e escola. Isso implica no afastamento de seus objetos pessoais, brinquedos, amigos e pessoas significativas.

A hospitalização pode representar uma ruptura com o meio social, atividades, hábitos e cultura da criança ${ }^{(16)}$. Portanto, a hospitalização pode provocar atraso ou interrupção do processo de desenvolvimento da criança $^{(20)}$. O hospital, em geral, não é organizado de forma a atender às individualidades das crianças, tendo seu enfoque no tratamento das enfermidades, se tornando para a criança um ambiente onde a dor se faz presente constantemente e onde ela recebe inúmeras restrições devido ao seu estado de saúde. Viver em ambiente adequado potencializa habilidades de linguagem, cognitiva e pessoal-social ${ }^{(21)}$.

Um dos estudos, ao observar que as crianças se interessavam por objetos próximos ao seu campo visual, indicou que o ambiente hospitalar deve conter estímulos visuais à criança, para que ela possa explorar o ambiente para além das grades de seu berço ou cama ${ }^{(20)}$. A exploração visual é relevante na integração da criança com o meio e serve como um estímulo para a exploração do ambiente.

O medo do desconhecido foi um dos fatores que dificultou a adaptação da criança à internação, sendo sugerido que se permita que a criança explore objetos desconhecidos com o tato, a fim de identificar os atributos e qualidades desses objetos ${ }^{(16,20)}$. O tato estimula o cérebro a reconstituir e multiplicar suas capacidades simbólicas. É importante que a criança hospitalizada se movimente, seja no próprio leito, seja pelos corredores. Os objetos e o espaço deixam de ser desconhecidos e imaginários, passando a ser reais e, assim, menos assustadores ${ }^{(20)}$.

Para que o ambiente hospitalar se torne menos ameaçador ao desenvolvimento saudável, sugeriu-se que a equipe de enfermagem proporcione um ambiente passível de exploração pela criança, onde haja possibilidade de aproximar o espaço hospitalar com o domiciliar, permitindo que a criança traga de casa objetos significantes como pelúcias e brinquedos ${ }^{(20)}$. Sugeriu-se ainda que os procedimentos sejam realizados com a participação da criança e família, pois o conhecimento sobre os procedimentos reduz o estresse e medo causados pelos $\operatorname{mesmos}^{(16)}$.

A compreensão e, sobretudo, a sensibilização da equipe de enfermagem pediátrica hospitalar, diante da importância desses aspectos, são fundamentais para a promoção de uma atenção humanizada e comprometida, que viabilize meios de amenização do sofrimento da criança durante o processo de hospitalização, bem como o impacto desse período sobre o seu desenvolvimento.

\section{Os inter-relacionamentos e o desenvolvimento infantil}

Os relacionamentos interpessoais são uma das principais fontes de estímulos para uma criança ${ }^{(20)}$. A interação com um adulto proporciona afeto, segurança, estimulação e aprendizagem, permitindo um desenvolvimento harmonioso, em âmbito emocional, psicomotor, lingüístico e cognitivo ${ }^{(20,22)}$.

Considerando a relevância da interação mãe-filho para o desenvolvimento saudável da criança, sendo que o início dessa relação se dá ainda no pré-natal, a construção dela deve ser incentivada pelo profissional de enfermagem. O estímulo à relação deve ser propiciado durante o pré-natal, no parto e pós-parto, oferecendo condições favoráveis para a construção afetiva entre mãe e filho ${ }^{(23)}$. Incentivar que a mãe dedique momentos do seu dia ao feto, proporcionar ambiente agradável durante o trabalho de parto e parto, promover atenção humanizada durante esses momentos, são medidas simples que proporcionam maior disponibilidade afetiva para que a mãe se dedique ao bebê que acaba de chegar ${ }^{(23)}$.

A formação de vínculo afetivo entre a criança e o cuidador, geralmente a mãe, assegura conforto, transmite segurança, satisfaz as funções fisiológicas de alimentação e oferece contato pele a pele e olho a olho, essenciais para a promoção de um desenvolvimento emocional saudável(23-24).

A equipe de enfermagem deve manter-se vigilante na qualidade de interação mãe-filho desde a maternidade, visto a importância dessa relação no desenvolvimento saudável da criança ${ }^{(23)}$. Um dos estudos incluídos nesta pesquisa demonstrou que, dentro do contexto hospitalar, pais e equipe de enfermagem muitas vezes falharam em prover holding satisfatório ${ }^{(24)}$. Outra pesquisa mostra que o nível de educação dos cuidadores está relacionado ao desenvolvimento de linguagem das crianças ${ }^{(21,22)}$. Estudos indicam a necessidade de um melhor preparo da equipe de enfermagem para atuar em pediatria, visto a especificidade desta clientela ${ }^{(16,20,23)}$.

O conceito de holding está relacionado há aspectos de intersubjetividade e de construção da relação com o outro, estando presente a habilidade de empatia, tão importante na produção de uma atuação de enfermagem holística e humanizada, fundamental na busca do estímulo ao desenvolvimento infantil.

Para estimular o desenvolvimento infantil, o ambiente deve oferecer um espaço físico adequado com objetos estimuladores. Cabe destacar que as pessoas que convivem com as crianças precisam ser estimuladoras, proporcionando experiências sensoriais, cognitivas, motoras e sociais à criança por meio do relacionamento interpessoal ${ }^{(16,20)}$. Momentos como o banho e a alimentação podem ser aproveitados para se tornarem estimuladores à criança ${ }^{(25)}$.

O relacionamento interconjugal aparece como um dos fatores que influenciam o desenvolvimento infantil. Pais que possuem um relacionamento saudável são mais propensos a se comportarem de maneira sensível a satisfazer as necessidades de seus filhos ${ }^{(26)}$. Enquanto que pais com relações conflituosas tendem ser emocionalmente mais esgotados e distraídos, menos atentos, coerentes e sensíveis às suas crianças. Crianças que vivem conflitos familiares são consideradas em situação de risco de desenvolvimento, quer pelo conflito em si, quer pelo comportamento parental que pode ser mais rígido e menos caloroso ${ }^{(26)}$.

Famílias que vivem conflitos interparceiros precisam de intervenções, não somente visando a resolução do conflito, mas 
também o aconselhamento sobre paternidade/maternidade e sua importância para o desenvolvimento social e comportamental da criança ${ }^{(26)}$. Compreender a relação entre conflito interparceiros e o desenvolvimento social e comportamental das crianças é um passo importante para as intervenções eficazes. Culturalmente, existe uma compreensão de que não se deve interferir em questões de relacionamentos familiares, fazendo com que o profissional de saúde comumente se abstenha desse tipo de atividade de aconselhamento em sua rotina de trabalho. É necessário que esses profissionais se sintam mais preparados para esse tipo de atuação.

Enfermeiros devem identificar quem é o membro familiar que toma as decisões para a família, especialmente em caso de cuidado à saúde, bem como a composição da família e de sua rede social, procurando conhecer quem são os principais membros estendidos ou não tradicionais das famílias, quais são as interações entre as pessoas, buscando apreender o significado delas para as crianças ${ }^{(27)}$. A identificação da rede e do apoio social possibilita conhecer valores e formas de interação que podem contribuir para o planejamento do cuidado à saúde da criança e sua família(27).

\section{CONSIDERAÇÕES FINAIS}

A partir dos estudos analisados é possível concluir que para prover condições para um desenvolvimento infantil adequado, se faz necessário que a enfermagem esteja atenta a diversos fatores aos quais as crianças estão expostas, seja pelo meio onde vivem ou por uma situação de doença, sofrimento ou hospitalização, ampliando o olhar sobre a criança para além das questões biológicas, oferecendo um cuidado integral e humanizado, tendo em vistas a criança no seu contexto social, cultural e familiar.

Nesse sentido, o lúdico é considerado essencial ao desenvolvimento infantil adequado, cabendo à equipe de enfermagem estimular sua utilização nos diversos contextos da vida da criança, inclusive no hospitalar. Aqui se destaca o potencial de utilização de algumas estratégias que devem ser exploradas pelo enfermeiro, como o Teatro Clown, as brinquedotecas, a arteterapia e a musicoterapia.

A fim de possibilitar a adaptação da criança ao ambiente hospitalar, a enfermagem deve proporcionar condições ambientais favoráveis à exploração, contendo estímulos visuais, sonoros e táteis. A integração da criança no espaço e a sua inclusão no processo terapêutico podem reduzir o trauma causado pela hospitalização e, dessa forma, reduzir o impacto sobre o desenvolvimento infantil.

Os relacionamentos interpessoais devem ser foco importante na vigilância do desenvolvimento infantil, considerando sua relevância diante de tal. O vínculo com a mãe ou cuidador e o relacionamento entre os pais são destacados como essenciais, principalmente para o desenvolvimento social e comportamental da criança. Conflitos nos relacionamentos familiares devem fazer parte das intervenções por parte dos profissionais de saúde, destacando-se aqui o papel do enfermeiro.

Entretanto, para que a enfermagem possa atuar desenvolvendo ações eficazes para promoção e estímulo do desenvolvimento infantil é imprescindível compreender as especificidades desta clientela e sua família, buscando ampliar os conhecimentos acerca do desenvolvimento infantil, suas alterações e normalidades, para assim estimular suas potencialidades.

\section{REFERÊNCIAS}

1. Programa das Nações Unidas para o Desenvolvimento [homepage na internet] Objetivos de Desenvolvimento do Milênio. [acesso em: 30 mar 2009]. Disponível em: www.pnud.org.br/odm.

2. Ministério da Saúde (Brasil). Saúde da Criança: acompanhamento do crescimento e desenvolvimento infantil. Normas e Manuais Técnicos. Brasília: Ministério da Saúde; 2002. (Cadernos de Atenção Básica n.11 - série A).

3. Organização Pan-americana da Saúde. Manual do Desenvolvimento Infantil no Contexto do AIDPI. Washington: OPAS; 2005.

4. Dessen MAC, Biasoli-Alves ZMM. O estudo da família como base para a promoção da tolerância. In: Biasoli-Alves ZMM, Fishmann R, organizadores. Crianças e adolescentes: construindo uma cultura da tolerância. São Paulo: Edusp; 2001; p. 183-193.

5. Halpern R. Poverty and early childhood parenting: toward a framework for intervention. Am J Orthopsychiatr 1990;60(1):6-18.

6. Cia F. Habilidades sociais parentais e o relacionamento entre pais e filho. Psico. Estud 2006;11(1): 73-81.
7. Bowlby J. Attachment and loss. Nova York: Basic Books; 1969.

8. Ainsworth M, Blehar M, Waters E, Wall S. Patterns of attachment: a psycological study of the strange situation. Hillsdale: Erlbaum; 1978.

9. Landry SH, Smith KE, Swank PR, Assel MA, Vellet S. Does early responsive parenting have a special importance for children's development or is consistency across early childhood necessary?. Dev Psychol 2001;37(3):387-403.

10. Mendes KDS, Silveira RCCP, Galvão CM. Revisão integrativa: método de pesquisa para a incorporação de evidências na saúde e na enfermagem. Texto \& Contexto Enferm 2008;17(4):58-64.

11. Galvão CM, Sawada NO, Trevizan, MA. Revisão sistemática: recurso que propicia a incorporação das evidências na prática de enfermagem. Rev Latinoam Enferm 2004;12(3):549-56.

12. Broome ME. Integrative literature reviews for the development of concepts. In: Rodgers BL, Knafl KA. Concept development in nursing: foundations, techniques and applications. 2. ed. Philadelphia: WB Saunders; 2000. p. 193-215. 
13. Paula CC de, Ravelli APX, Zinn LR, Motta MGC. Cuidado de enfermagem na aventura do desenvolvimento infantil: reflexões sobre o lúdico no mundo da criança. Cogitare Enferm 2002;7(2): 30-4.

14. Lima RAG, Azevedo EFA, Nascimento LC, Rocha SMM. A arte do teatro clown no cuidado às crianças hospitalizadas. Rev Esc Enferm USP 2009;43(1):186-93.

15. Ravelli APX, Motta MGC. O lúdico e o desenvolvimento infantil: um enfoque na música e no cuidado de enfermagem. Rev Bras Enferm 2005;58(5):611-3.

16. Magnabosco G, Tonelli ALNF, Souza SNDH. Abordagens no cuidado de enfermagem à criança hospitalizada submetida a procedimentos: uma revisão de literatura. Cogitare Enferm 2008;13(1):103-8.

17. Valladares ACA, Carvalho AMP. A arteterapia no contexto da hospitalização pediátrica. O desenvolvimento da construção com sucata hospitalar. Acta Paul Enferm 2005; 18(1):64-71.

18. Valladares ACA, Carvalho AMP. Promoção de habilidades gráficas no contexto da hospitalização. Rev. Eletrônica Enferm 2006;8(1):128-33.

19. Stouffer JW, Shirk BJ, Polomano RC. Practice guidelines for music interventions with hospitalized pediatric patients. J Pediatr Nurs 2007;22(6):448-56.

20. Bortolote GS, Brêtas JRS. O ambiente estimulador ao desenvolvimento da criança hospitalizada. Rev Esc Enferm USP 2008;42(3):422-9.
21. Rezende MA, Beteli VC, Santos JLF. Avaliação das habilidades de linguagem e pessoal sociais pelo teste de Denver II em instituições de educação infantil. Acta Paul Enferm 2005;18(1):56-63.

22. Rezende MA, Lima FG, Beteli VC, Santos JLF. Habilidades de linguagem e pessoal social de crianças de 0 a 3 anos de idades cuidadas em creches. Rev Bras Crescimento Desenvolv Hum 2003;13(1): 40-52.

23. Silva LR da, Chiristoffel MM, Fernández AM, Santos IMM dos. A importância da interação mãe-bebê no desenvolvimento infantil: a atuação da enfermagem materno-infantil. Rev Enferm UERJ 2006;14(4): 606-12.

24. Regis FC, Kakehashi TY, Pinheiro EM. Análise do cuidado ao bebê hospitalizado segundo a perspectiva winnicottiana. Rev Bras Enferm 2005;58(1):39-43.

25. Harrison LL, Williams AK, Berbaum ML, Stem JT, Leeper J. Effects of developmental, health status, behavioral, and environmental variables on preterm infants' responses to a gentle human touch intervention. Int J Prenatal Perinatal Psychol Med 2000;12(1):109-122.

26. Whiteside-Mansell L, Bradley RH, McKelvey L, Fussell JJ. Parenting: linking impacts of interpartner conflict to preschool children's social behavior. J Pediatr Nurs 2009;24(5):389-400.

27. Nehring WM. Cultural considerations for children with intellectual and developmental disabilities. J Pediatr Nurs 2007;22(2):93-102. 